\title{
Sets of Points Self-Conjugate with regard to a Quadric in $n$ Dimensions
}

\author{
By D. M. Y. Sommerville, \\ Victoria University College, Wellington, New Zealand. \\ (Received 28th March, 1931. Read 6th November, 1931.)
}

$\S 1$. In space of three dimensions the properties of self-conjugate tetrads, pentads and hexads with regard to a quadric are well known (see Baker's Principles of Geometry, vol. iii). The general theorem in space of $n$ dimensions $S_{n}$ is to establish the existence of a set of $n+p+1$ points $A_{0}, A_{1}, \ldots, A_{n+i}(0 \leqslant p \leqslant n-1)$ such that the pole, with respect to a given quadric, of the $(n-1)$-flat determined by any set of $n$ of the points lies in the $p$-flat determined by the remaining $p+1$ points.

$\S 2$. Consider a space $S_{n+p}$ containing $S_{n}$, and $n+p+1$ linearly independent points $A_{0}^{\prime}, A_{1}^{\prime}, \ldots, A_{n+p}^{\prime}$. Then there is a quadric $Q^{\prime}$ in $S_{n+i}$ with respect to which the points form a self-polar simplex. If $p=0$ this simplex forms the self-conjugate set of $n+1$ points whose existence is in question. If $p>0$, let $S_{p-1}$ be the polar $(p-1)$-flat of $S_{n}$ with respect to $Q^{\prime}$, and project the figure on to $S_{n}$ with $S_{p-1}$ as axis of projection. The process is as follows. To project a point $A^{\prime}$ : determine the $p$-flat through $A^{\prime}$ and $S_{\mathrm{p}-1}$; this cuts $S_{n}$ in the corresponding point $A$. Generally, to project an $r$-flat $R^{\prime}$ : determine the $(p+r)$-flat through $R^{\prime}$ and $S_{p-1}$; this cuts $S_{n}$ in the corresponding $r$-flat $R$. To project the quadric $Q^{\prime}:$ an $(n+p-1)$-flat through $S_{p-1}$ and touching the quadric has $n-1$ degrees of freedom and cuts $S_{i}$ in an $(n-1)$-flat which envelopes the corresponding quadric $Q$ in $S_{n}$. In the present case, since $S_{p-1}$ is the polar of $S_{n}, Q$ is actually the section of $Q^{\prime}$ by $S_{n}$. The assemblage of $(n+p-1)$ flats through $S_{p-1}$ envelopes a hypercone of species $p$ having $S_{p-1}$ as vertex-edge; this is a tangent hypercone to $Q^{\prime}$, and the points of contact form the quadric $Q$.

We proceed to show that the $n+p+1$ points $A_{r}$ obtained in this way form a self-conjugate set with respect to the quadric $Q$, i.e. that the pole, with respect to $Q$, of the $(n-1)$-flat $a$ determined by any set of $n$ of the points $A_{0}, \ldots, A_{n-1}$ lies in the $p$-flat $\beta$ determined by the remaining $p+1$ points $A_{n}, \ldots, A_{n \div p}$. 
The corresponding $n$ points $A_{0}^{\prime}, \ldots, A_{n-1}^{\prime}$ determine an $(n-1)$-flat $a^{\prime}$, and this determines with $S_{p-1}$ an $(n+p-1)$-flat whose pole with respect to $Q^{\prime}$ lies in $S_{n}$ and also in the $p$-flat $\beta^{\prime} \equiv\left(A_{n}^{\prime}, \ldots, A_{n+p}^{\prime}\right) . \quad P$ is therefore the point of intersection of $S_{n}$ with $\beta^{\prime}$. Now the corresponding $p$-flat $\beta \equiv\left(A_{n}, \ldots, A_{n+p}\right)$ is the intersection of $S_{n}$ with the (2p)-flat determined by $S_{p-1}$ and $\beta^{\prime}$; hence $P$ lies in $\beta$. (It is necessary that $2 p<n+p$, and therefore $p<n$ ). Also since $P$ is conjugate, with respect to $Q^{\prime}$, to every point in the $(n+p-1)$-flat $\left(a^{\prime}, S_{p-1}\right)$, it is conjugate to every point in the section of this by $S_{n}$. But this section is $a$, and the section of $Q^{\prime}$ by $S_{n}$ is $Q$; hence $P$ is the pole of a with respect to $Q$, and it has been proved also that $P$ lies in $\beta$.

It follows further that the polar $r$-flat $(0 \leqslant r<n-p)$ of the $(n-r-1)$-flat determined by any set of $n-r$ of the points $\left(A_{0}, \ldots, A_{n-r-1}\right)$ lies in the $(p+r)$-flat determined by the remaining $p+r+1$ points $\left(A_{n-i}, \ldots, A_{n+p}\right)$.

$\S 3$. If the simplex $A_{0}^{\prime}, \ldots, A_{n+i}^{\prime}$ is taken as frame of reference, the tangential equation of the quadric $Q^{\prime}$ is of the form

$$
\xi_{0}^{2}+\ldots+\xi_{n+p}^{2}=0 .
$$

Any linear equation in $\left(\xi_{r}\right)$ represents a point $P^{\prime}$ in $S_{n+j}$. The point $A^{\prime}{ }_{r}$ is represented by the equation $\xi_{r}=0 . \quad S_{p-1}$ is represented by $p$ linear equations $\Sigma_{1}=0, \ldots, \Sigma_{j}=0$ in $\xi_{0}, \ldots, \xi_{n+p}$. Any linear equation in $\left(\xi_{r}\right)$, together with the $p$ equations $\Sigma_{p}=0$, represents the assemblage of $(n+p-1)$-flats through $S_{p-1}$ and the point $P^{\prime}$; these cut $S_{n}$ in an assemblage of $(n-1)$-flats all passing through the corresponding point $P$. The quadratic equation $\Sigma \xi_{r}^{2}=0$, together with the $p$ equations $\Sigma_{p}=0$, represents the assemblage of $(n+p-1)$ flats through $S_{p-1}$ and touching $Q^{\prime}$; these cut $S_{n}$ in an assemblage of $(n-1)$-flats all touching $Q$.

Hence in $S_{n}$ the equation

$$
\xi_{0}^{2}+\ldots+\xi_{n+p}^{2}=0
$$

where $\xi_{r}$ are connected by $p$ linear equations $\Sigma_{p}=0$, represents a quadric $Q$, and the equation $\xi_{r}=0$ represents the point $A_{r}$. The self-conjugate set of $n+p+1$ points $A_{r}$ is thus related to the representation of the quadric by a tangential equation in terms of $n+p+1$ squares.

$\S 4$. The reciprocal relations are at once deduced. When a quadric $Q$ in $S_{i}$ is represented by a point-equation

$$
x_{0}^{2}+\ldots+x_{n+p}^{2}=0
$$


in terms of $n+p+1$ squares, the variables $x_{r}$ being connected by $p$ linear equations $S_{p}=0$, the $n+p+1$ primes, or $(n-1)$-flats, $x_{r}=0$ form a self-conjugate set such that the polar prime with respect to $Q$ of the point common to any $n$ of the primes passes through the $(n-p-1)$-flat common to the remaining $p+1$ primes; and so on.

§5. If $S$ and $\Sigma^{\prime}$ are two quadrics such that a simplex exists which is inscribed in $S$ and self-polar with respect to $\Sigma^{\prime}$ then an infinity of such simplexes exists, and $S$ is said to be outpolar to $\Sigma^{\prime}$.

If the point-equation of $S$ is

$$
S \equiv \sum \sum a_{r s} x_{r} x_{s}=0
$$

and the tangential equation of $\Sigma^{\prime}$ is

$$
\Sigma^{\prime} \equiv \Sigma \Sigma A_{r 8}^{\prime} \xi_{r} \xi_{s}=0
$$

the condition that $S$ should be outpolar to $\Sigma^{\prime}$ is the vanishing of the bilinear invariant

$$
\Theta^{\prime} \equiv \Sigma \sum a_{r s} A_{r s}^{\prime}=0 .
$$

These relations still hold when $S$ is a cone of any species. Let $S$ be a cone in $S_{n}$ whose vertex-edge $S_{1,-1}$ is the $(p-1)$-flat determined by the vertices $A_{0}, \ldots, A_{p-1}$ of the simplex of reference. Its equation is then a homogeneous quadratic containing the variables $x_{p}, \ldots, x_{n}$ alone, i.e., in equation (1), $a_{r s}=0$ if $r$ or $s<p$.

Let $S_{n-p}$ denote the polar $(n-p)$-flat of $S_{p-1}$ with respect to $\Sigma^{\prime}$. We may choose the frame of reference so that $S_{n-p}$ is represented by the equations $x_{0}=0, \ldots, x_{p-1}=0$. Then the point-equation of $\Sigma^{\prime}$ is

$$
S^{\prime} \equiv \Sigma \Sigma a_{r s}^{\prime} x_{r} x_{\varepsilon}=0
$$

where $a_{r s}^{\prime}=0$ if $r<p$ and $s>p-1$ or vice versa.

Consider the sections $C$ and $C^{\prime}$ of $S$ and $S^{\prime}$ by $S_{n-p} . \quad C$ is represented by

$$
\Sigma \Sigma a_{r s} x_{r} x_{s}=0, \quad x_{0}=0, \ldots, x_{p^{\prime-1}}=0,
$$

the first equation being precisely the same as (1).

$C^{\prime}$ is represented by

$$
\Sigma \Sigma a_{r s}^{\prime} x_{r} x_{s}=0, \quad x_{0}=0, \ldots, x_{p-1}=0,
$$

and in the first equation we may now assume $a_{r s}^{\prime}=0$ if $r$ or $s<p$. (5) and (8) then represent two quadrics $C$ and $C^{\prime}$ in $S_{n-p}$ in terms of the coordinates $x_{p}, \ldots, x_{n}$. 
The determinant of $S^{\prime}$ is

and that of $C^{\prime}$ is

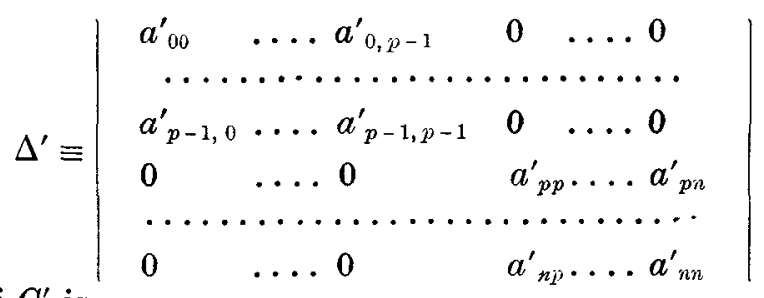

$$
\delta^{\prime}=\left|\begin{array}{c}
a_{p p}^{\prime} \ldots \ldots a_{p n}^{\prime} \\
\ldots \ldots \ldots \\
a_{n p}^{\prime} \ldots \ldots a_{n n}^{\prime}
\end{array}\right| .
$$

$A_{r s}^{\prime}$ is the cofactor of $a_{r s}^{\prime}$ in $\Delta^{\prime}$; let $a_{r s}^{\prime}$ be the cofactor of $a_{r s}^{\prime}$ in $\delta^{\prime}$. Then if

we have

$$
D \equiv\left|\begin{array}{c}
a_{00}^{\prime} \ldots \ldots a_{0, p-1}^{\prime} \\
\ldots \ldots \ldots \\
a_{p-1,0}^{\prime} \ldots \ldots \\
a_{p-1, p-1}^{\prime}
\end{array}\right|
$$

$$
A_{r s}^{\prime}=D a_{r s}^{\prime}
$$

when $r$ and $s$ are each greater than $p-1$.

When the cone $S$ is outpolar to the quadric $\Sigma^{\prime}$, we have

$$
\Sigma \Sigma a_{r s} A_{r s}^{\prime}=0
$$

the summations extending from $p$ to $n$. Hence from (7)

$$
\Sigma \Sigma a_{r s} a_{r s}^{\prime}=0
$$

Therefore the quadric $C$ is outpolar to the quadric $C^{\prime}$.

Hence if $S$ is a cone with vertex-edge $S_{p-1}, \Sigma^{\prime}$ any quadric, and $S_{n-p}$ the polar of $S_{p-1}$ with respect to $\Sigma^{\prime}$, then if $S$ is outpolar to $\Sigma^{\prime}$ the section of $S$ by $S_{n-p}$ is outpolar to the section of $\Sigma^{\prime}$.

§. Returning now to the simplex $A_{0}^{\prime}, \ldots, A_{n+p}^{\prime}$ in $S_{n+p}$ and its projection $A_{0}, \ldots, A_{n+p}$ on the $n$-flat $S_{n}$, and the quadric $Q^{\prime}$ for which $A_{0}^{\prime}, \ldots, A_{n+p}^{\prime}$ is self-polar, $S_{p-1}$ being the polar of $S_{n}$ with respect to $Q^{\prime}$, let $R^{\prime}$ be a cone with vertex-edge $S_{p-1}$ and passing through the $n+p+1$ points $A_{r}{ }_{r}$. $R^{\prime}$ is thus outpolar to $Q^{\prime}$. Let $R$ be the section of $R^{\prime}$ by $S_{n}$, and $Q$ the section of $Q^{\prime}$. Then $R$ is outpolar to $Q$ and contains the $n+p+1$ points $A_{0}, \ldots, A_{n+p}$ which form a self-conjugate set with respect to $Q$.

Hence if $\Sigma^{\prime}$ and $S$ are two quadrics in $S_{n}$ such that there exists $a$ set of $n+p+1$ points $(0 \leqslant p \leqslant n-1)$ inscribed in $S$ and selfconjugate with respect to $\Sigma^{\prime}, S$ is oulpolar to $\Sigma^{\prime}$. The existence of a simplex inscribed in $S$ and self-polar with respect to $\Sigma^{\prime}$ thus implies also the existence of a self-conjugate $r$-ad $(n+1 \leqslant r \leqslant 2 n)$ similarly inscribed. 